\title{
Ethische Reflexion und Entscheidungsfindung im professionellen Pflegehandeln realisieren
}

\author{
Annette Riedel
}

Online publiziert: 22. Dezember 2012

(C) Springer-Verlag Berlin Heidelberg 2012

Handlungsfeldübergreifend besteht der Wunsch und das Bestreben der professionell Pflegenden, eine qualitätvolle, am Individuum orientierte Pflege zu realisieren, die den aktuellen pflegewissenschaftlichen Erkenntnissen entspricht. Die professionelle Pflege verfolgt den Anspruch, theoretischen Anforderungen und individuellen Bedürfnissen gerecht zu werden, was nicht selten zu Spannungsfeldern führt. Pflegebezogene Handlungssituationen sind geprägt von verschiedenen Lebenswelten, Lebensentwürfen und Lebenskontexten, von Asymmetrien und Diversitäten. Sie werden beeinflusst von subjektiven Betroffenheiten und von Widersprüchen, die für die Beteiligten auf der Basis ihrer persönlichen Werteorientierung Wahrheit und Gültigkeit beanspruchen. Das heißt, Pflegesituationen werden in ihrer situativen Einzigartigkeit von unterschiedlichen - nicht immer kongruenten Werten - geleitet und beeinflusst. Die sich aus der Wertepluralität ergebenden Dilemmata fordern pflegeethisch begründete Positionierungen. Ethische Fragestellungen sind folglich ein genuiner Teil der Pflegepraxis, die ethische Dimension des pflegeberuflichen Auftrags ist unstrittig ([1, 2, 8]; [10], S. 39-59). Die vorhandenen pflegeethischen Kompetenzen sind demgegenüber nicht immer ausreichend, um die moralischen Irritationen zu analysieren und auszudrücken, um eine verantwortungsvolle, ethisch reflektierte und pflegeprofessionell begründete Positionierung darzulegen und diese gegenüber den Beteiligten zu vertreten. Für die Pflegenden ist es oftmals diffizil, die Komplexität und Vielschichtigkeit der jeweiligen ethischen Entscheidungsbedarfe zu explizieren.

Bewusst geht es im Folgenden um die ethische Reflexion im Pflegehandeln. Die hierfür erforderlichen Kompetenzen stellen die Grundlage dar, ethisch zu argumentieren, sich als professionell Pflegende in systematisierte Formen der ethischen Entscheidungsfindung einzubringen und die Perspektive der Profession Pflege im interdisziplinären ethischen Diskurs zu vertreten. Ethische Reflexion ist als genuiner Gegenstand professionellen Pflegehandelns eine Handlungsanforderung, die einen gleichwertigen Stellenwert zur Pflegediagnostik ver-

Prof. Dr. A. Riedel $(\bowtie)$

Vorstandsmitglied der Akademie für Ethik in der Medizin e. V.,

Fakultät Soziale Arbeit, Gesundheit und Pflege, Hochschule Esslingen,

Flandernstr. 101, 73732 Esslingen, Deutschland

E-Mail: annette.riedel@hs-esslingen.de 
dient. Ethische Reflexion hat das Ziel, dem umfänglichen Anspruch an eine individuumsund werteorientierte Pflege gerecht zu werden und beide Gesichtspunkte sukzessive im professionellen Handeln miteinander zu verbinden [8].

Im Kontext von Werteorientierung geht es nicht alleine um die Anerkennung von Werten und die Festlegung einer Verhaltenspraxis beziehungsweise Handlungsnorm. Werteorientierung kann nicht mit Werteübernahme, Werteerziehung, Wertemanagement oder einer (weiteren) spezifischen Form pflegeberuflicher Normbildung gleichgesetzt werden. Das würde bezogen auf den hier zugrunde liegenden Anspruch zu kurz greifen. Vielmehr geht es darum, Werteorientierung in Verbindung mit einer differenzierten Werteanalyse, einer reflektierten Werteentwicklung und einer bedachten Werteumsetzung zu begreifen, dies mit dem Ziel, die ethisch-moralische Handlungskompetenz zu vertiefen und einzufordern ([8], S. 202). Werteorientierung ist eine professionelle Haltung, die repräsentiert, was dem Handeln „Gründe, Kriterien und damit Orientierung“ verleiht ([4], S. 149). Werteorientierung fordert demnach von den Pflegenden einen bewusst eingenommenen wertebezogenen Standpunkt, den es argumentativ zu vertreten gilt. Werteorientierung kann so verstanden auch professionsinterne - vornehmlich pflegefachlich und/oder rechtlich orientierte - Normen und Regelwerke in Frage stellen beziehungsweise hinterfragen (Leitlinien, Standards, Qualitätskriterien, Grundprinzipien, Regeln etc.). Werteorientierung wirkt nicht einer standardgemäßen vielmehr aber einer unreflektierten standardisierten Versorgung entgegen! Sich des Einflusses und der Bedeutsamkeit der Werteorientierung beständig bewusst zu sein, stellt hohe Anforderungen an die pflegebezogenen Aushandlungsprozesse und die Ausgestaltung von Pflegesituationen ([2], S. 157, 168, 169). Im Pflegealltag heißt das: Die einzelfallbezogene Situationsspezifik erhält neben der Individuumsorientierung eine weitere Perspektive. Bei der gezielten Werteorientierung werden analysierte beteiligte Werte (professionelle und subjektive) in das Reflexions- und Handlungsfeld pflegeberuflicher Praxis integriert: Mit der ethischen Reflexion wird die Individuumsorientierung ergänzt und die berufliche Perspektive um ein wesentliches Element erweitert [8].

Ethische Reflexion trägt dazu bei, über bestehende Vorgaben hinauszuschauen, sie will hinterfragen, die pflegefachliche Perspektive erweitern. Sie fordert, die Verhältnisse zwischen den beteiligten Werten und Werteorientierungen zu explizieren und zu ordnen $[8,10]$. Es geht hier nicht um einen zu standardisierenden Prozess, sondern um eine bewusste und intendierte Analyse der pflegerelevanten Situation mit der Perspektive auf deren moralischen Gehalt sowie auf die beteiligten Werte und die Werteorientierungen der Beteiligten.

Die ethische Reflexion ist so verstanden grundlegend für die Erfassung des Ethikfokus und die Formulierung der situativen ethischen Fragestellung. Ethische Reflexion verfolgt nicht den Anspruch, ethisch begründete Entscheidungen zu treffen, sondern „,darüber nachzudenken, auf welchen Grundannahmen und Vorverständnissen die Probleme beruhen“ ([5], S. 59), sie erlaubt zunächst „mehr Distanz zu dem Geschehen“ ([7], S. 31).

An dieser Stelle wird exemplarisch eine Forderung an die Profession - die im Expertenstandard Ernährungsmanagement [3] formuliert wird - aufgegriffen, um zu explizieren, was die Realisierung der geforderten Standardkriterien an vorausgehenden Schritten und grundlegenden Kompetenzen verlangt. So wird auf der Strukturebene (S 2a), bezogen auf die Pflegefachkraft, formuliert: „Kompetenz zur Entscheidungsfindung bei ethisch komplexen Fragestellungen.“ Unter den Ergebniskriterien (E2) ist zu lesen: „Die [...] Maßnahmen sind [...] ethisch begründet" ([3], S.31).

Vor der Realisierung der in dem Expertenstandard geforderten Entscheidungsfindung im Rahmen einer „Fallbesprechung“ ([3], S. 40, 41) - müssen indes folgende Schritte der ethischen Reflexion vorausgehen: 
1. Die Uneinigkeiten darüber, was in spezifischen (Pflege-)Situationen moralisch gut und richtig ist, aufgreifen, moralische Irritationen bewusst wahrnehmen, diese in Worte fassen und kommunizieren (Identifikation).

2. Die Irritationen auf ihren moralischen Gehalt hin analysieren und die ethische Fragestellung in Abgrenzung zu rein fachlichen oder rein organisatorischen Fragestellungen herausstellen [9] (Analyse).

3. Die Bedeutsamkeit der Werteorientierung ergänzend zur vertrauten Individuumsorientierung erfassen, die situative ethische Dimension darlegen und die Relevanz der ethischen Entscheidungsfindung vertreten (Explikation).

Zum aktuellen Zeitpunkt ist zunächst bei der ethischen Reflexion anzusetzen, sowohl in der Ausbildung am Lernort Praxis als auch im Kontext von Fort- und Weiterbildung. Parallel zu der Frage nach dem pflegefachlich korrekten und evidenzbasierten Handeln sind Anleitungssituationen, Pflegeprozessplanungen und Pflegevisiten bewusst um folgende Fragen zu ergänzen: Gibt es moralische Irritationen in der Situation? Welche Werte sind in der Situation beteiligt? Wessen (professionsbezogene) Wertevorstellungen leiten die Handlungsentscheide? Welche Werteorientierungen prägen das Handeln? etc. ([2], S. 157, 168, 169; [9]). Das heißt, parallel zur Individuumsorientierung und zur Evidenzorientierung erhalten ethische Reflexion, Wertereflexion und die bewusste Werteorientierung einen angemessenen Stellenwert und werden somit zu einem ultimativen Gegenstand pflegeprofessioneller Handlungen und Entscheidungsprozesse. An dieser Stelle geht es noch nicht darum, ein ethisches Dilemma zu bearbeiten oder zu provozieren, sondern um die Sensibilisierung und die Realisierung ethischer Reflexion und Werteorientierung im Praxisalltag, um eine unerlässliche Perspektivenerweiterung und eine damit einhergehende Kompetenzentwicklung. Das heißt für die Pflegepraxis, zunächst die in den o. g. drei Schritten implizierten Kompetenzen zu stärken, als so genannte „Grundkompetenzen“ ethischer Reflexion: Die Sensibilität in Bezug auf moralische Irritationen und die Kompetenz, diese zu identifizieren, die Kompetenz, das spezifisch „Ethische“ innerhalb der (Pflege-)Situation zu analysieren und auszuweisen, die Kompetenz, den Bedarf ethischer Entscheidungsfindung darzulegen und zu vertreten. Hierbei geht es nicht darum, die ethische Fragestellung alleine beantworten zu können, sondern darum, die ethische Fragestellung mit ihren Spezifika benennen zu können. Dies ist grundlegend dafür, sich als professionell Pflegende(r) in den Prozess der ethischen Entscheidungsfindung konstruktiv und ethisch argumentativ - aus der spezifischen Perspektive der professionellen Pflege - einbringen zu können und bei ethisch reflektierten und verantwortungsvollen Entscheidungen mitzuwirken. Sind diese Kompetenzen und Handlungsvollzüge im Pflegealltag gestärkt, ist pflegeethische Reflexion im Praxisalltag implementiert, gilt es, die systematisierten Formen der ethischen Reflexion und Entscheidungsfindung einzufordern, dann, wenn ein ethisches Dilemma als solches erkannt und erfasst wird. Möglicherweise führt die oben beschriebene Sensibilisierung und bewusst praktizierte Werteorientierung in der Pflegepraxis zunächst zu einem Anstieg pflegeethischer Fragestellungen. Dies sollte als „Gewinn“ bewertet werden, da es Folgendes zeigt: Die Pflegenden sind sensibel für moralische Irritationen, erkennen und identifizieren ethische Fragestellungen/DilemmaSituationen. Entscheidend ist, dass sie den Mut haben und ermutigt werden, diese zu benennen. Fortschreitend wird die Bedeutsamkeit ethischer Reflexion bewusst, die direkt in den nächsten Schritt überleitet: das strukturierte Umgehen, die systematische Entscheidungsfindung im Kontext ethischer Fragestellungen. Erst dann kann die Forderung der Expertenstandards realisiert werden: „Entscheidungsfindung bei ethisch komplexen Fragestellungen“ ([3], S.31). 
Die Forderungen an eine sukzessive und praxisorientierte Heranführung der professionell Pflegenden an (interdisziplinäre) ethische Entscheidungsfindungsprozesse führt zu Management-Herausforderungen an die Einrichtungen und ambulanten Dienste, verbunden mit der konkreten Aufforderung aktiv zu werden und die entsprechenden Rahmenbedingungen zu schaffen. Die Stärkung der ethischen Reflexion und Werteorientierung, der Weg hin zu einer systematisierten und strukturierten ethischen Entscheidungsfindung in den Einrichtungen der Altenhilfe, der Behindertenhilfe, in Hospizen und der ambulanten Pflege sollte jetzt aus Profession und Institution heraus entwickelt, etabliert und sukzessive als Gegenstand professionellen Pflegehandelns konsolidiert werden. Das heißt: Jetzt, aus der Überzeugung heraus, dass ethische Reflexion und Ethikberatung vor dem Hintergrund der strukturellen Komplexität und der zunehmenden ,ethischen Komplexität“ ([6], S. 26) in der Pflegepraxis unverzichtbar ist. Jetzt, als Impuls aus der Praxis heraus, dass ethische Reflexion und Werteorientierung einen wesentlichen Beitrag zu einer qualitätvollen Pflege leistet und somit unabdingbar ist. Jetzt, aus der Erkenntnis heraus, dass die Qualität, Akzeptanz und Transparenz der ethisch reflektierten Entscheidungsfindung durch ein strukturiertes und systematisiertes Verfahren verdichtet werden kann. Jetzt, aus der Erfahrung heraus, dass ethische Reflexion und ethische Fallbesprechungen zu einer erheblichen Entlastung der Mitarbeiter(innen) führen und somit obligat sind. Jetzt, aus dem professionellen Verständnis heraus, dass dadurch die Profession gestärkt und die Professionalität sichtbar wird. Somit lautet die Forderung: Ethische Reflexion und Ethikberatung jetzt motiviert und an dem pflegeberuflichen Auftrag orientiert aus der Praxis heraus verwirklichen und etablieren, bevor der MDK und/oder die Heimaufsichtsbehörden Ethikberatung postulieren und in die Praxis hineinprüfen.

\section{Literatur}

1. Bobbert M (2011) Ethik in der Pflege. In: Stoecker R, Neuhäuser C, Raters M-L (Hrsg) Handbuch Angewandte Ethik. J.B. Metzler, Stuttgart, S 403-406

2. Bobbert M (2011) Die Pflege nicht entscheidungsfähiger Patienten und die Ausweitung des Autonomiekonzeptes. In: Breitsameter C (Hrsg) Autonomie und Stellvertretung in der Medizin. Entscheidungsfindung bei nichteinwilligungsfähigen Patienten. W. Kohlhammer, Stuttgart, S 139-173

3. Deutsches Netzwerk für Qualitätsentwicklung in der Pflege (Hrsg) (2010) Expertenstandard Ernährungsmanagement zur Sicherstellung und Förderung der oralen Ernährung in der Pflege. Schriftenreihe des Deutschen Netzwerks für Qualitätsentwicklung in der Pflege, Osnabrück

4. Feeser-Lichterfeld U, Heyer M (2010) Handeln verantworten lernen. Anspruch und Möglichkeit ethischer Bildung. In: Baranzke H et al (Hrsg) Handeln verantworten. Grundlagen - Kriterien - Kompetenzen. Herder, Freiburg i. Br., S 145-173

5. Maio G (2010) Zur Orientierungslosigkeit einer Medizin ohne ethische Reflexion. In: Heinemann W, Maio G (Hrsg) Ethik in Strukturen bringen. Denkanstöße zur Ethikberatung im Gesundheitswesen. Herder, Freiburg i. Br., S 59-77

6. Monteverde S (2012) Das Umfeld pflegeethischer Reflexion. In: Monteverde S (Hrsg) Handbuch Pflegeethik. Ethisch denken und handeln in den Praxisfeldern der Pflege. W. Kohlhammer, Stuttgart, S $19-41$

7. Rabe M (2012) Ethische Reflexion und Entscheidungsfindung in der intensivmedizinischen Praxis. In: Salomon F (Hrsg) Praxisbuch Ethik in der Intensivmedizin. Konkrete Entscheidungshilfen in Grenzsituationen. 2., aktualisierte und erweiterte Auflage. Medizinische Wissenschaftliche Verlagsgesellschaft, Berlin, S 29-39

8. Riedel A (2012) Werteorientierung - Relevanz der Akzentuierung in der professionellen Pflege. Z Evang Ethik 56:197-206

9. Riedel A, Lehmeyer S (2012) Professionelles Handeln ethisch reflektieren - Ethische Fragestellungen erkennen und benennen. Forum Ausbild 6:30-34

10. Riedel A, Lehmeyer S (2013) Konzeptentwicklung: Theoretische Fundierung und Prämissen zur Konzeptualisierung ethischer Fallbesprechungen. In: Riedel A, Lehmeyer S, Elsbernd A. Einführung von ethischen Fallbesprechungen - Ein Konzept für die Pflegepraxis. Ethisch begründetes Handeln praktizieren. 3., aktualisierte und überarbeitete Aufl. Jacobs, Lage, S 39-157 